\title{
Corrigendum: Interrogation of genomes by molecular copy-number counting (MCC)
}

Angelika Daser, Madan Thangavelu, Richard Pannell, Alan Forster, Louise Sparrow, Grace Chung, Paul H Dear \& Terence H Rabbitts Nat. Methods 3, 447-453 (2006); published online 23 May 2006; corrected after print 8 June 2006.

Correspondence should be addressed to M. Thangavelu (mt370@hutchison-mrc.cam.ac.uk) instead of T. H. Rabbitts. The error has been 